\title{
Cerium Reduction at the Interface between Ceria and Yttria-stabilised Zirconia and Implications for Interfacial Oxygen Non-stoichiometry
}

\author{
Kepeng Song ${ }^{1,2}$, Herbert Schmid ${ }^{3}$, Vesna Srot ${ }^{1}$, Elisa Gilardi ${ }^{4}$, Giuliano Gregori ${ }^{4}$, Kui Du $^{2}$, Joachim \\ Maier ${ }^{4}$, Peter A. van Aken ${ }^{1}$ \\ 1. Max Planck Institute for Intelligent Systems, Stuttgart, Germany \\ 2. Shenyang National Laboratory for Materials Science, Chinese Academy of Sciences, Shenyang, China \\ 3. INM-Leibniz Institute for New Materials, Saarbrücken, Germany \\ 4. Max Planck Institute for Solid State Research, Stuttgart, Germany
}

$\mathrm{CeO}_{2}$ and $\mathrm{Y}_{2} \mathrm{O}_{3}$-stabilized zirconia (YSZ) are two typical candidates for electrolyte materials in solid oxide fuel cells attributed to their high ion conductivities. However, the conductivities of these materials are closely connected with high temperatures. Therefore, it is important to develop electrolyte materials which can conduct oxygen ions at lower temperatures. Recent experiments have shown that hetero-structures consisting of alternating layers of Gd-doped $\mathrm{CeO}_{2}$ and YSZ exhibit higher ionic conductivity compared to either of the bulk materials [1]. However, the mechanism for the high ionic conductivity of these hetero-structures is still unclear. Theoretical calculations indicate that the oxygen vacancy formation energy is considerably reduced at the interfaces and oxygen vacancies expected to segregate to the interfaces might provide highways for rapid ion conduction [2]. The aim of our work is to obtain insights into the structure and chemistry of interfaces between $\mathrm{CeO}_{2}$ and YSZ by scanning transmission electron microscopy (STEM) combined with electron energy-loss spectroscopy (EELS).

Epitaxial $\mathrm{CeO}_{2}$ films were grown on YSZ (111) substrates using pulsed laser deposition. TEM samples in cross-sectional geometry were prepared by the tripod polishing technique followed by $\mathrm{Ar}^{+}$ion-milling while cooling the specimen with liquid nitrogen. Reduction of cerium ions at the interface between $\mathrm{CeO}_{2}$ films and YSZ substrates is demonstrated using aberration-corrected scanning transmission electron microscopy combined with electron energy-loss spectroscopy. Investigations were carried out at $200 \mathrm{kV}$ in an analytical TEM/STEM microscope (JEOL-ARM 200F) equipped with cold field-emission gun (C-FEG) and a CEOS probe Cs-corrector. A post-column imaging energy-filter (GATAN GIF Quantum ER) attached to this system is used for EELS and electron spectroscopic imaging.

A bright-field (BF) STEM image of the cross-sectional view is presented in Figure 1a, showing the $\mathrm{CeO}_{2} / \mathrm{YSZ}$ interface. The $\mathrm{CeO}_{2}$ film is approximately $30 \mathrm{~nm}$ thick and continuous. Figure $1 \mathrm{~b}$ presents a selected area electron diffraction pattern from zone axis $\left\langle 1-10>_{\mathrm{YSZ}}\right.$ showing that the $\mathrm{CeO}_{2}$ film and YSZ substrate have a cubic on cubic orientation relationship $\left((111)<1-10>_{\mathrm{CeO} 2} / /(111)<1-10>_{\mathrm{YSZ}}\right)$. No reaction layers or other phases can be observed at the interface. Periodical misfit dislocations were observed at the interface with extra atomic planes appearing in YSZ (Figure 1c and 1d). It is well known that the $\mathrm{Ce}-\mathrm{M}_{4,5}$ edges are valence sensitive. Since the possible presence of $\mathrm{Ce}^{3+}$ is seen as evidence of oxygen vacancy formation, oxygen states of cerium ions near the interface were investigated by EELS. Intensity ratios of $\mathrm{Ce}-\mathrm{M}_{4,5}$ white-lines were used to determine the valence state of cerium. Measured spectra were compared with known reference spectra acquired from compounds containing $\mathrm{Ce}^{3+}$ or $\mathrm{Ce}^{4+}$. In addition, quantitative analysis has been performed on the $\mathrm{Ce}-\mathrm{M}_{4,5}$ edges to study the ratio of $\mathrm{Ce}^{3+}$ to $\mathrm{Ce}^{4+}$ as function of the distance from the interfaces. It is revealed that most of the Ce ions were reduced from $\mathrm{Ce}^{4+}$ to $\mathrm{Ce}^{3+}$ at the interface region with a decay of several nanometers. Several 
possibilities of charge compensations are discussed. Irrespective of the details, such local non-stoichiometries are crucial not only for understanding charge transport in such hetero-structures but also for understanding ceria catalytic properties $[3,4]$.

\section{References:}

[1] S. Azad, O.A. Marina, C.M. Wang, L. Saraf, V. Shutthanandan, D.E. McCready, A. El-Azab, J.E. Jaffe, M.H. Engelhard, C.H.F. Peden, S. Thevuthasan, Applied Physics Letters 86 (2005) 131906.

[2] M. Fronzi, S. Cereda, Y. Tateyama, A. De Vita, E. Traversa, Physical Review B 86 (2012) 085407. [3] K. Song, H. Schmid, V. Srot, E. Gilardi, G. Gregori, K. Du, J. Maier, P.A. van Aken, submitted to APL Materials (2014).

[4] The authors acknowledge funding from the PhD student exchange program between the Max Planck Society and the Chinese Academy of Sciences and the Natural Sciences Foundation of China (Grant No. 51221264). The research leading to these results has received funding from the European Union Seventh Framework Program [FP/2007-2013] under grant agreement no 312483 (ESTEEM2).

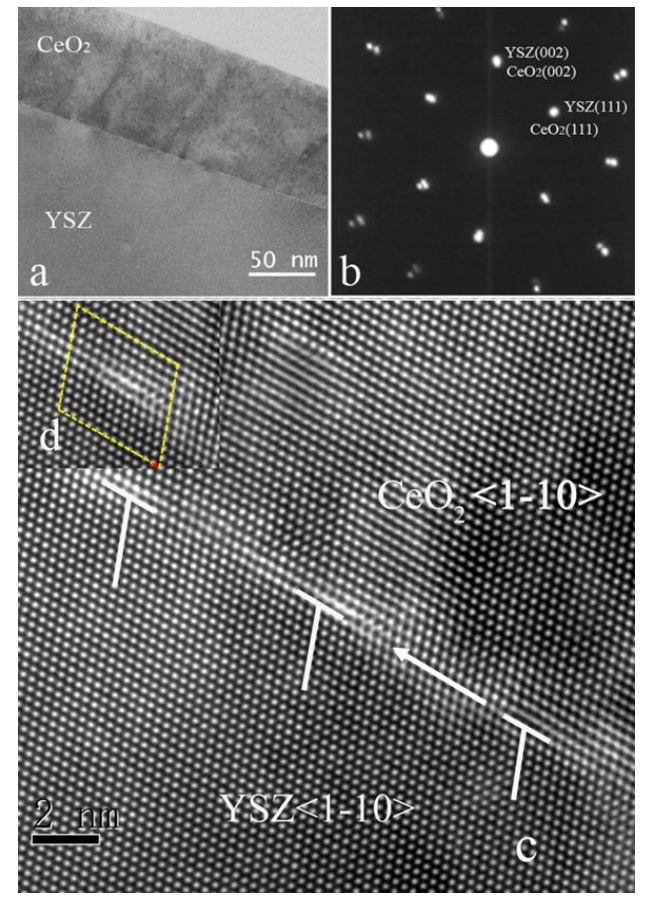

Figure 1. Morphology of the film shown by the low magnification BF-STEM image (a) and orientation relationship revealed by selected area electron diffraction patterns (b) from zone axis $<1-10>_{\text {YSZ }}$. (c) HRTEM micrograph of the $\mathrm{CeO}_{2} / \mathrm{YSZ}$ (111) interface viewed from the [1-10]YSZ direction; the position of the interface is indicated by an arrow. (d) Inset displays a detail of the interface showing the Burgers circuit of the misfit dislocations.

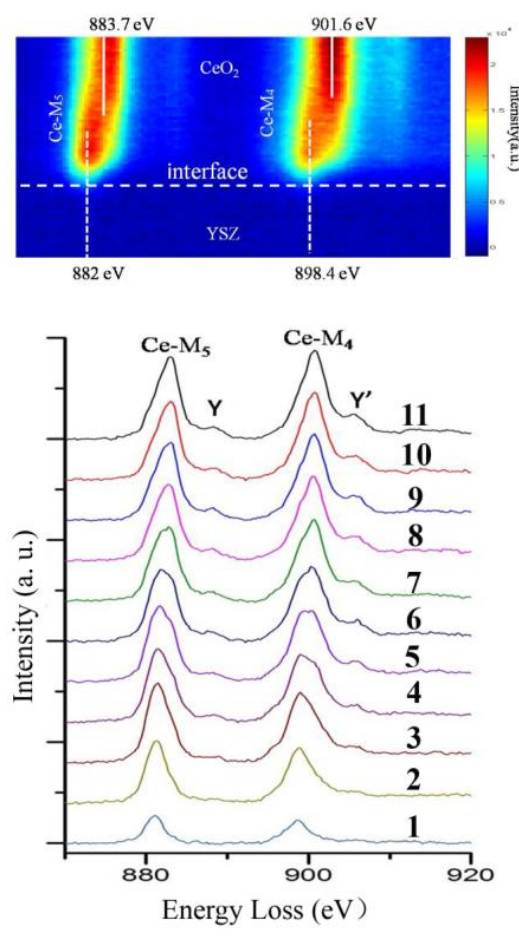

Figure 2. EELS spectrum image from a line scan (top) with $\mathrm{Ce}-\mathrm{M}_{4,5}$ spectra (bottom) extracted from the spectrum image, which illustrates the change of $\mathrm{Ce}-\mathrm{M}_{4,5}$ edge from the bulk (spectrum 11) to the interface (spectrum 1). 